•综述・

\title{
基于现代物种共存理论的入侵生态学概念框架
}

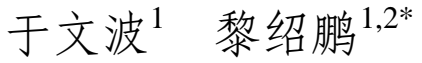 \\ 1 (华东师范大学生态与环境科学学院, 浙江天童森林生态系统国家野外科学观测研究站, 上海 200241) \\ 2 (崇明生态研究院, 上海 202162)
}

摘要: 在入侵生态学 60 多年的发展历程中, 生态学家提出了多种多样的假说来解释生物入侵的机制。这些纷繁复 杂的假说在丰富我们对生物入侵认知的同时, 也给入侵生态学概念的整合带来了困难。其中, 外来种和土著种是 否存在生态学差异, 以及这种差异如何影响生物入侵, 是入侵生态学研究和争论的焦点问题。现代物种共存理论 通过将外来种和土著种的生态学差异划分为生态位差异和适合度差异, 为入侵生态学概念的整合提供了新的视 角。依据该理论, 外来种可以通过两种策略实现成功入侵: 一是扩大与土著种的生态位差异, 二是提高自身相较于 土著种的适合度优势。因此, 外来种-土著种的生态位差异和适合度差异共同决定了入侵的成败与危害程度。通过 对经典入侵假说进行梳理, 我们发现大部分假说都可以在该理论框架下进行解读, 不同假说的主要差别在于强调 不同生态学过程对生态位和适合度差异的影响。同时, 这一理论框架很好地解释了为什么外来种-土著种的亲缘关 系和性状差异会对生物入侵产生复杂的影响, 为达尔文归化谜团的和解以及外来种-土著种功能性状的比较研究 提供了新的思路。目前, 现代物种共存理论还处于快速发展的阶段, 依旧存在很多不足, 但将其运用到生物入侵的 实证研究中将是入侵生态学今后一个重要的发展方向。

关键词: 生态位; 竞争能力; 入侵机制; 达尔文归化谜团; 功能性状

\section{Modern coexistence theory as a framework for invasion ecology}

\author{
Wenbo $\mathrm{Yu}^{1}$, Shaopeng $\mathrm{Li}^{1,2^{*}}$ \\ 1 Zhejiang Tiantong Forest Ecosystem National Observation and Research Station, School of Ecological and \\ Environmental Sciences, East China Normal University, Shanghai 200241 \\ 2 Institute of Eco-Chongming, Shanghai 202162
}

\begin{abstract}
In the 60-year development of invasion ecology, many hypotheses have been proposed to explain the mechanisms behind biological invasion. However, it remains difficult to integrate these hypotheses into a unified framework. In particular, whether exotics ecologically differ from natives, and how ecological differences between exotic and native species could determine invasion outcome, have been hotly debated. By categorizing exotic-native ecological differences into niche differences and fitness differences, modern coexistence theory provides a framework to place different invasion hypotheses and mechanisms into a common context. This framework emphasizes that invasion success depends on either a niche difference between exotics and natives, or that exotics have fitness advantage over natives. By reviewing the leading invasion hypotheses, we found that most invasion hypotheses can be incorporated into this framework, as they considered different aspects of exotic-native niche and fitness differences. This framework can well explain why exotic-native phylogenetic distance and trait difference have complex influences on invasion, and therefore may help to reconcile the long-standing Darwin's naturalization conundrum and the debate regarding the value of native versus exotic trait comparisons. Together, this framework provides a new opportunity to better understand the mechanisms of ecological invasion.
\end{abstract}

Key words: niche; competitive ability; invasion mechanisms; Darwin’s naturalization conundrum; functional traits 
哪些外来种更容易入侵? 哪些生境更容易被 入侵? 我们如何应对和管理生物入侵? 这三个问题 一直是入侵生态学研究的核心内容。围绕这三个问 题, 入侵生态学经历了漫长而曲折的发展历程。早 在19世纪, 达尔文在On the Origin of Species就已经 探讨了外来种成功定居和归化的决定因素(Darwin, 1859)。但直到100年后“动物生态学之父” Charles Elton 的 The Ecology of Invasion by Animals and Plants一书的出版(Elton, 1958), 才标志着入侵生态 学真正的开端。但这一著作在当时并未引起学术界 的足够重视。直到20世纪80年代中期, 随着环境问 题科学委员会(the Scientific Committee on Problems of the Environment, SCOPE)这一国际组织的建立和 一系列关于生物入侵的专著的出版(如Mooney \& Drake, 1986; Drake et al, 1989), 入侵生态学才真正 进入了快速发展时期。一方面, 入侵生态学的学科 体系和理论框架得到了进一步的确立(Williamson, 1996; Shigesada \& Kawasaki, 1997; 郑景明和马克 平, 2010; 万方浩等, 2015); 另一方面, 解释生物入 侵机制的假说不断被提出和完善(徐承远等, 2001; 徐汝梅和叶万辉, 2003; Lockwood et al, 2007; 王明 娜等, 2014, 吴昊和丁建清, 2014)。我国的入侵生态 学研究在近20年中蓬勃发展, 在生物入侵的格局、 机制和控制等领域取得了若干重要进展, 为入侵生 态学理论的发展做出了重要贡献(鞠瑞亭等, 2012)。

在入侵生态学快速发展的同时, 也伴随着一些 质疑和争论。一方面, 以Mark Davis为代表的生态 学家认为不应该以原产地来评判一个物种, 外来种 和土著种并没有实质性的差别, 生物入侵可以整合 入群落生态学的研究体系之中, 入侵生物学作为一 门学科应该被终结(Davis, 2009; Davis et al, 2011; Thompson \& Davis, 2011; Valéry et al, 2013)。这一观 点引起了以Daniel Simberloff为代表的生态学家的 强力反驳, 他们指出外来种和土著种在进化历史、 与人类活动的相关性、对生物多样性的影响和潜在 危害等方面均具有显著差异性, 忽视这种差异会带 来重大的科学和政策风险(Pyšek \& Hulme, 2009; Simberloff, 2011, 2015; Richardson \& Ricciardi, 2013; Simberloff \& Vitule, 2014)。Daniel Simberloff的观点 得到了绝大部分生态学家的支持, 而外来种和土著 种生态学差异性的比较逐渐成为入侵生态学研究 的核心科学问题(van Kleunen et al, 2010a)。

另一方面, 随着入侵生态学的快速发展, 生态 学家提出了众多的假说来解释外来种成功入侵的 机制。我们可以列出一个长长的生物入侵假说清单, 比如Catford等(2009)曾列举和归纳了29个生物入侵 假说。这些假说相互独立又彼此关联, 但是没有一 个假说是完美的。每种假说只能解释特定外来种和 入侵地的生物入侵, 却对其他外来种和入侵地的情 形无能为力。我们很难用一个统一的框架将这些假 说尽可能简单地组织起来, 这给初学者进入这一研 究领域带来了很大困难。正如Lawton (1999)感叹群 落生态学是一团糟一样, 如何整合入侵生态学数量 繁多而又相互混杂的假说是我们面临的严峻挑战。 为实现这一整合, 生态学家从各个角度进行了尝 试。例如Richardson和Pyšek (2006)从外来种入侵性 和土著群落可入侵性的角度对已有的假说进行了 梳理和整合，Catford等(2009)将不同入侵假说按照 繁殖体压力、非生物因素、生物因素以及人类活动 对这些因素的影响进行了划分，而Blackburn等 (2011)从入侵阶段和每个阶段的屏障出发, 尝试建 立了一个统一的生物入侵概念框架。这些尝试有力 地促进了入侵理论的整合, 但也存在一些不足。其 中一方面就是这些框架侧重于假说和入侵阶段的 划分, 较少梳理众多假说之间内在的逻辑联系。近 20年来, 现代物种共存理论引起了生态学家越来越 多的关注, 并逐渐被引入到入侵生态学的研究之 中。由于该理论综合考虑了外来种的入侵性和土著 群落的可入侵性, 并聚焦外来种和土著种的生态学 差异这一核心问题，为整合不同入侵假说提供了一 个新的思路。

\section{基于现代物种共存理论的入侵生态学㮛}

\section{1 经典物种共存理论与生物入侵}

生物入侵在一定程度上可以看成外来种和土 著种共存的问题。长期以来，以生态位分化为核心 的经典物种共存理论在生物入侵的研究中一直占 据主导地位(Levine \& D’Antonio, 1999)。这一理论 强调外来种和土著种生态位的差异是入侵成功与 否的关键。例如外来种可以通过占据未被土著种占 据的生态位而实现成功入侵(空余生态位假说, empty niche hypothesis; Elton, 1958); 生物多样性低 的群落更容易被入侵，因为其留给外来种的空余生 
态位和资源更多(生物抵抗假说, biotic resistance hypothesis; Elton, 1958)。但是基于生态位理论的概 念框架也存在一些不足: 一是很难解释和土著种占 据相似生态位的外来种为何可以成功入侵; 二是在 解释外来种的成功入侵和危害程度上存在一定矛 盾。如果外来种通过和土著种的生态位分化实现成 功入侵, 那么它们和土著种的竞争作用应该很弱, 这无法解释为什么一些成功入侵的外来种会对土 著种产生严重危害。现代物种共存理论为调和这一 矛盾提供了一个可行的思路。

\section{2 现代物种共存理论}

现代物种共存理论由Peter Chesson (2000)系统 性地提出, 是对经典物种共存理论的一次重要拓展 和革新。储诚进等(2017)对该理论的定义、内涵、 实证和应用等进行了系统的介绍。简单而言, 现代 物种共存理论将物种间的差异分为两类: 生态位差 异和适合度差异。其中生态位差异的定义与经典物 种共存理论近似, 强调的是物种在资源、空间、时 间和天敌等维度上的分化。例如深根系和浅根系的 植物、生活在湖泊表层和底层的鱼类、利用不同碳 源的微生物, 均具有较高的生态位差异。物种之间 的生态位差异越大, 种间竞争的强度越小, 因此越 容易共存。适合度差异强调的是物种在资源利用效 率、对抗天敌取食和生长繁殖能力上的差异, 可以 通俗地理解为物种竞争能力的差异。适合度差异越 大的物种越难以共存, 因为适合度高的物种总是倾 向于将适合度低的物种竞争排除出去。

基于此, 在一个群落中物种可以通过两种机制 实现共存。一是提高彼此间的生态位差异, 避免生 态位的重叠, 进而实现稳定共存。这一机制被称为 稳定化机制(stabilizing mechanisms)。二是降低彼此 间的适合度差异, 当两个物种的竞争能力旗鼓相当 时, 也就很难将对方竞争排除出去。该机制被称为 均等化机制(equalizing mechanisms)。因此, 在一个 现实的群落中, 物种能否稳定共存取决于生态位差 异和适合度差异的相对大小(Chu \& Adler, 2015)。其 中, 中性理论(neutral theory)假定物种既没有生态 位差异也没有适合度差异, 因此可以看作是现代物 种共存理论的一个特例(Adler et al, 2007)。由于现代 物种共存理论可以很好地调和生态位理论和中性 理论之间的矛盾, 并逐渐将Janzen-Connell效应、植 物-土壤反馈(plant-soil feedback)、天敌逃逸(enemy release)、种间正相互作用(facilitation)、优先级效应 (priority effects) 等生态学重要概念整合其中 (Chesson, 2018; Ellner et al, 2019; Grainger et al, 2019a; Song et al, 2019; Ke \& Wan, 2020), 目前该理 论已经成为生态学最受关注的理论热点之一。

\section{3 基于现代物种共存理论的入侵生态学概念框架}

基于现代物种共存理论，MacDougall等(2009) 提出了一个入侵生态学的概念框架。该框架从外来 种-土著种生态位差异和适合度差异的角度阐述了 生物入侵成功和危害的决定机制(图1)。首先，外来 种和土著种的生态位差异有助于外来种的成功入 侵，但会降低外来种对土著种的危害程度。这是由 于生态位差异降低了外来种和土著种的种间竞争, 促进了双方的共存。因此，和土著种生态位差异大 的外来种往往容易入侵成功，但其危害程度相对较 小。其次，外来种和土著种的适合度差异是具有等 级(hierarchical)和方向性的。当外来种的适合度高于 土著种时，二者的适合度差异越大，外来种越容易 入侵成功，并对土著种造成的危害更大; 而当外来 种的适合度低于土著种时, 二者的适合度差异越大， 外来种越难入侵成功, 其危害程度也就越低。简单 而言, 相比土著种, 外来种的适合度优势越大, 即其 竞争能力越强时，越容易入侵成功并造成更大危害。

基于该框架，外来种可以采用两种“策略”实现 成功入侵。一是尽可能地提高与土著种的生态位差 异，实现生态位分化，降低竞争强度，从而实现和 土著种的共存，执行这一“策略”的外来种通常具有 较低的危害性。二是尽可能提高自身的适合度，在 和土著种的竞争中占据优势，进而“战胜”土著种， 执行这一“策略”的外来种通常具有较高的危害性。 因此，在群落中危害程度高的外来种往往是那些和 土著种生态位差异小但适合度高的物种。总体而言, 对于任何一个现实的群落, 外来种和土著种的生态 位差异和适合度差异的大小共同决定了入侵的成 败及其对土著群落的危害程度。

\section{2 入侵生态学若干重要假说的理论整會}

外来种-土著种的生态位差异和适合度差异同 时影响着外来种的入侵能力和土著群落的可入侵 性，并贯穿整个入侵阶段。不同生态学过程均可通 过作用于这两种差异来影响生物入侵。因此，现代 


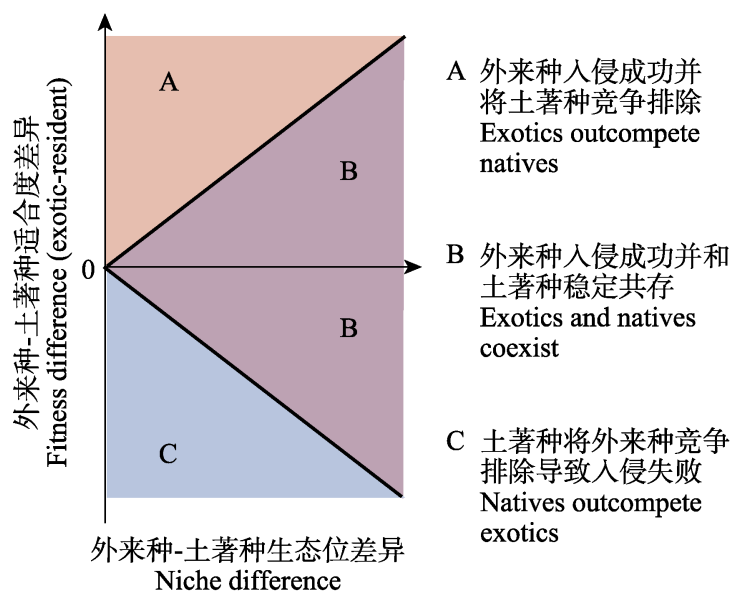

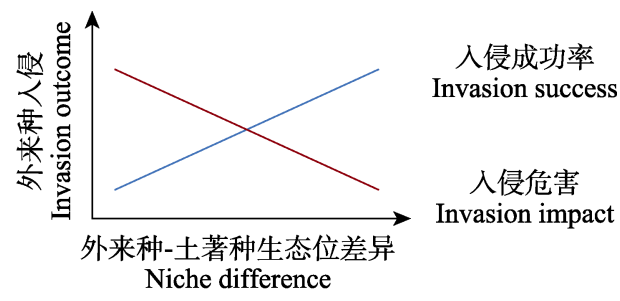

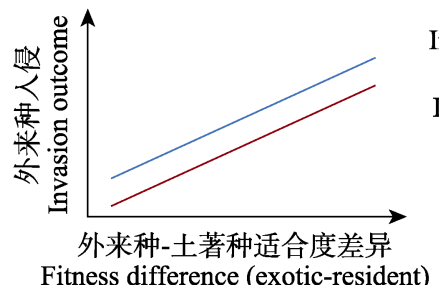

人侵成功率

Invasion success

人侵危害

Invasion impact

图1 基于现代物种共存理论的入侵生态学概念框架。该框架认为入侵的成功和危害取决于外来种-土著种的生态位差异和适 合度差异。其中外来种-土著种的生态位差异促进生物入侵但会降低入侵危害, 而外来种相对于土著种的适合度优势会同时 促进生物入侵和加剧危害程度。修改自MacDougall等(2009)、Cadotte等(2018)和Li等(2019)。

Fig. 1 A conceptual diagram using modern coexistence theory as a framework for invasive ecology. According to the framework, the invasion outcome depends on both exotic-native niche and fitness differences. On one hand, increasing exotics-native niche difference promotes the success of the exotics but hinders their impacts on natives. On the other hand, fitness difference, here measured as the fitness advantage of invaders over the natives, could enhance both invasion success and their impacts on native species. Adapted from MacDougall et al (2009), Cadotte et al (2018), and Li et al (2019).

物种共存理论为整合生物入侵的各种假说提供了 一个思路。通过对经典入侵假说进行梳理, 我们发 现大部分入侵假说可以在这个统一框架下展开进 一步的解读和阐述(表1)。其中部分假说侧重生态位 差异, 部分假说侧重适合度差异, 很少有假说同时 考虑了两个方面的差异。

\section{1 外来种-土著种生态位差异相关假说}

与外来种-土著种生态位差异相关的入侵假说 可以分为两类, 一类强调外来种和土著种本身的生 态位差异, 另一类则强调外部环境和物种自身的变 化对生态位差异的改变(表1)。第一类假说, 如空余 生态位假说、生物抵抗假说、资源机遇假说(resource opportunity hypothesis)、环境异质性假说 (environmental heterogeneity hypothesis)等, 均强调 外来种通过占据未被土著种占据的生态位来实现 成功入侵。第二类假说, 如干扰假说 (disturbance hypothesis)、波动资源假说 (fluctuating resources hypothesis) 以及和全球变化相关的假说, 大多强调 的是由于人类活动或自然原因导致的环境变化为 外来种提供了适合的生态位, 进而促进了其成功入 侵。同时, 外来种也可以通过表型可塑性和适应性 进化, 扩大自身与土著种的生态位差异, 提高成功 入侵的可能性(Pettitt-Wade et al, 2015)。综合起来, 尽管不同假说强调了外来种-土著种生态位差异形
成的不同机制, 但无论从外来种的入侵性还是土著 群落的可入侵性而言, 这些假说均与“外来种-土著 种生态位差异促进生物入侵”这一现代物种共存理 论的预测一致。由于这类假说聚焦生态位差异, 通 常忽略适合度差异, 可以很好地解释和土著种差异 大的外来种的入侵机制, 但在解释和土著种差异小 的外来种(如姐妹种, sister species)的入侵机制及危 害时, 则比较困难。

\section{2 外来种-土著种适合度差异相关假说}

同聚焦于生态位差异的假说一致, 和外来种土著种适合度差异相关的入侵假说也可以分为两 类: 一类强调外来种相较于土著种具有更高的适合 度; 另一类则强调外部环境和物种自身的变化导致 入侵过程中外来种适合度的提高(表1)。第一类假说, 如内禀优势假说(inherent superiority hypothesis)、理 想杂草特征假说(ideal weed characteristics hypothesis)和繁殖体压力假说 (propagule pressure hypothesis)等强调外来种本身在形态、生理、生态、 遗传和行为等方面具备先天优势, 或者通过拥有较 多繁殖体数量等方式, 导致外来种具有更高的适合 度, 进而实现成功入侵。第二类假说, 如竞争能力 增强进化假说 (evolution of increased competitive ability)、氮分 配进化假说 (hypothesis of the evolution of nitrogen allocation)和新武器假说(new weapon 
表1 主要生物入侵假说及其在现代物种共存理论框架下的解读

Table 1 Leading invasion hypotheses and their relationship to modern coexistence theory

\begin{tabular}{|c|c|c|c|c|}
\hline $\begin{array}{l}\text { 假说 } \\
\text { Hypothesis }\end{array}$ & $\begin{array}{l}\text { 定义 } \\
\text { Definition }\end{array}$ & $\begin{array}{l}\text { 强调的差异 } \\
\text { Ecological } \\
\text { difference }\end{array}$ & $\begin{array}{l}\text { 在现代物种共存理论框架下的解读 } \\
\text { Interpretation under the framework of } \\
\text { modern coexistence theory }\end{array}$ & $\begin{array}{l}\text { 参考文献 } \\
\text { References }\end{array}$ \\
\hline $\begin{array}{l}\text { :余生态位假说 } \\
\text { mpty niche } \\
\text { pothesis }\end{array}$ & $\begin{array}{l}\text { 外来种通过占领入侵地未被土著种占据的空余生 } \\
\text { 态位, 进而实现成功入侵。Exotics successfully } \\
\text { invade when occupying niche space unused by the } \\
\text { native communities. }\end{array}$ & $\begin{array}{l}\text { 生态位差异 } \\
\text { Niche } \\
\text { difference }\end{array}$ & $\begin{array}{l}\text { 外来种和土著种的生态位差异导致空余生 } \\
\text { 态位的存在, 促进入侵。Exotic-native niche } \\
\text { differences create empty niches and promote } \\
\text { invasion. }\end{array}$ & $\begin{array}{l}\text { Elton, } \\
1958\end{array}$ \\
\hline
\end{tabular}

生物抵抗假说 多样性高的群落能够更有效地利用资源, 空余的生 生态位差异 多样性高的群落土著种和外来种生态位重 Elton, Biotic resistance 态位更少, 因此更难以被入侵。More diverse Niche 叠的概率更高, 抑制入侵。Higher 1958 hypothesis communities have less available resources and vacant difference niches, preventing invasion. probability of exotic-native niche overlap in more diverse communities inhibits invasion.

资源机遇假说

Resource

opportunity

hypothesis

入侵地具备外来种必需的生物和非生物资源, 且这 生态位差异 些资源大多未被土著种有效利用，促进外来种入 Niche

侵。The resident communities are more susceptible to difference invasions when they contain high availability of resources on which a potential invader depends.

环境异质性假说 异质性高的生境中具有更多的生态位，外来种可以 生态位差异 Environmental

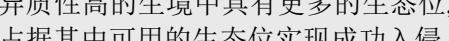
占据其中可用的生态位实现成功入侵。

Niche

heterogeneity

Heterogeneous environment contain a diverse array of difference hypothesis niches, and invaders would be successful by filling the available niches.

干扰假说

Disturbance

干扰在群落中形成空余生态位，降低土著群落的抵

hypothesis

\section{波动资源假说 \\ Fluctuating \\ resources \\ hypothesis}

抗力, 促进外来种成功定居。Disturbance events increase vacant niches and decrease the resistance of native communities, promoting invasion.

资源在时间上的波动打破了资源供给和获取的平 衡, 导致外来种可利用资源的增加, 提高了群落的 可入侵性。Fluctuations in resource availability change resource input and uptake rates, and promote invasion.
外来种和土著种的生态位差异导致入侵地 中外来种的必需资源未被利用, 促进入侵。 Exotic-native niche differences making the essential resources of exotics available, promoting invasion.

在异质性高的环境中, 外来种和土著种的 Melbourne 生态位差异大, 促进入侵。Exotic-native et al, 2007 niche differences would be larger in heterogeneous environment, promoting invasion.

生态位差异 Niche difference

干扰导致空余生态位的存在, 促进了和土 著种生态位差异大的外来种的入侵。Empty MacIsaac, niches created by disturbance events benefit 1997 the invasion of exotics with large niche differences to natives.

生态位差异 资源的波动提供了空余生态位，促进了和 Davis et al, Niche 土著种生态位差异大的外来种的入侵。

difference Fluctuating resources offer empty niches, which benefit the invasion of exotics with large niche differences to natives.

生态位差异 远缘物种生态位差异大, 因此与土著种亲 Niche 缘关系远的外来种更容易入侵。Distantly related species have large niche differences, which promote the invasion of exotics that distantly related to natives.

naturalization hypothesis

尔文认为和本土物种亲缘关系远的外来种 , 因为它们和本土物种差异大, 生态位 are more likely to naturalize, as they occupy distinct niches and complete less stronger with natives.

内禀优势假说

Inherent superiority hypothesis

成功入侵的外来种在形态、生理、生态、遗传和行 为等特征上具备独特的内禀优势。Successfully Fitness invaded exotics have intrinsic characteristics superior difference to native species.

理想杂草特征假 说 Ideal weed characteristics 入侵种具备类似理想杂草的生物特性，这些特征使 适合度差异 其很好地适应环境, 并在竞争中占据优势。Invasive Fitness species share similar characteristics to ideal weeds, making them well adapt to the environment and exhibit competitive advantages.

繁殖体压力假说 繁殖体引入的数量、频率和质量在入侵初期起决定 Propagule pressure hypothesis 性作用，繁殖体压力越大，成功入侵概率越高。 High quantity, frequency and quality of propagule introductions increase chance of successful invasion.

适合度差异 Fitness difference

相比土著种, 成功入侵的外来种本身具有

Lowry et 更高适合度。Successfully invaded exotics are al, 2013 at a fitness advantage relative to the resident communities.

相比土著种, 成功入侵的外来种具有的杂 草特征使其具有更高的适合度。Exotic species with ideal weed characteristics show higher fitness than the native species in the resident communities.

繁殖体压力越大, 外来种适合度越高, 越容 Lockwood 易入侵成功。Exotic species with high et al, 2005 propagule pressure show high fitness advantage, and are more likely to invade.

适合度差异

Fitness 化假说 Evolution 敌的物质和能量转移到生长系统, 增强自身竞争能 of increased competitive 力, 实现成功入侵。After releasing from native specialist predators, exotic species evolve with less investment in anti-herbivore defenses and allocate more resources to vegetative and reproductive growth. difference 竞争能力的增强使外来种具有更高的适合 度, 进而在和土著种竞争时占据优势, 成功 Notzold, 入侵。The increased competitive ability 1995 ability This strategy promotes invasion. makes the exotics show higher fitness invasion. 
表 1 (续) Table 1 (continued)

\begin{tabular}{|c|c|c|c|c|}
\hline $\begin{array}{l}\text { 假说 } \\
\text { Hypothesis }\end{array}$ & $\begin{array}{l}\text { 定义 } \\
\text { Definition }\end{array}$ & $\begin{array}{l}\text { 强调的差异 } \\
\text { Ecological } \\
\text { difference }\end{array}$ & $\begin{array}{l}\text { 在现代物种共存理论框架下的解读 } \\
\text { Interpretation under the framework of } \\
\text { modern coexistence theory }\end{array}$ & $\begin{array}{l}\text { 参考文献 } \\
\text { References }\end{array}$ \\
\hline $\begin{array}{l}\text { 氮分配进化假说 } \\
\text { Hypothesis of the } \\
\text { evolution of } \\
\text { nitrogen } \\
\text { allocation }\end{array}$ & $\begin{array}{l}\text { 相比原产地, 外来种在入侵地降低体内氮元素向防 } \\
\text { 御作用的分配, 增加了氮向光合机构的转移, 提高 } \\
\text { 了自身光合能力。Comparing to native ranges, exotics } \\
\text { evolve to decrease nitrogen allocation to defenses and } \\
\text { increase nitrogen allocation to photosynthesis in } \\
\text { introduced ranges. }\end{array}$ & $\begin{array}{l}\text { 适合度差异 } \\
\text { Fitness } \\
\text { difference }\end{array}$ & $\begin{array}{l}\text { 氮分配的改变提高了外来种在入侵地的适 } \\
\text { 合度。The evolution of nitrogen allocation } \\
\text { increases the fitness of exotics in the } \\
\text { introduced habitats. }\end{array}$ & $\begin{array}{l}\text { Feng et al, } \\
2009\end{array}$ \\
\hline $\begin{array}{l}\text { 新武器假说 New } \\
\text { weapon } \\
\text { hypothesis }\end{array}$ & $\begin{array}{l}\text { 入侵植物能够分泌特异的化感物质抑制土著种。土 } \\
\text { 著种对这些化感物质缺乏适应性, 导致外来种在竞 } \\
\text { 争中占据上风。Exotics release allopatric chemicals } \\
\text { that inhibit and repress the native competitors in new } \\
\text { range, enhancing the exotics' competitive ability and } \\
\text { success. }\end{array}$ & $\begin{array}{l}\text { 适合度差异 } \\
\text { Fitness } \\
\text { difference }\end{array}$ & $\begin{array}{l}\text { 化感物质提高了外来种的适合度, 降低了 } \\
\text { 土著种的适合度, 促进入侵。Allopatric } \\
\text { chemicals increase the fitness of exotics and } \\
\text { decrease the fitness of natives, promoting } \\
\text { invasion. }\end{array}$ & $\begin{array}{l}\text { Callaway \& } \\
\text { Aschehoug, } \\
2000\end{array}$ \\
\hline $\begin{array}{l}\text { 天敌逃逸假说 } \\
\text { Enemy release } \\
\text { hypothesis }\end{array}$ & $\begin{array}{l}\text { 当外来种被引入入侵地后, 脱离了原产地天敌的控 } \\
\text { 制, 进而可以快速生长繁殖扩散。After introduction } \\
\text { into new areas, exotics escape from their old enemies, } \\
\text { benefiting their growth and spread. }\end{array}$ & $\begin{array}{l}\text { 适合度差异 } \\
\text { Fitness } \\
\text { difference }\end{array}$ & $\begin{array}{l}\text { 脱离天敌控制后, 外来种适合度增加, 提 } \\
\text { 高了相对于土著种的适合度优势, 有利于 } \\
\text { 成功入侵。After escaping from the natural } \\
\text { enemies, exotics increase their fitness, } \\
\text { promoting invasion. }\end{array}$ & $\begin{array}{l}\text { Keane \& } \\
\text { Crawley, } \\
2002\end{array}$ \\
\hline $\begin{array}{l}\text { 预适应假说 } \\
\text { Preadaptation } \\
\text { hypothesis }\end{array}$ & $\begin{array}{l}\text { 达尔文认为近缘种偏好相似的生境, 因此土著种占 } \\
\text { 据的生境更适合与其近缘的外来种定居。Close } \\
\text { relatives favor similar environments, the habitats } \\
\text { occupied by native species were more suitable for the } \\
\text { establishment of their closely related exotics. }\end{array}$ & $\begin{array}{l}\text { 适合度差异 } \\
\text { Fitness } \\
\text { difference }\end{array}$ & $\begin{array}{l}\text { 在被土著种占据的生境中, 与土著种亲缘 } \\
\text { 关系近的外来种具有更高适合度, 因此更 } \\
\text { 容易入侵。In the habitats occupied by native } \\
\text { species, the exotics that closely related to } \\
\text { these natives would have high fitness and are } \\
\text { more likely to invade. }\end{array}$ & $\begin{array}{l}\text { Ricciardi \& } \\
\text { Mottiar, } \\
2006\end{array}$ \\
\hline $\begin{array}{l}\text { 新生态位假说 } \\
\text { Novel niche } \\
\text { hypothesis }\end{array}$ & $\begin{array}{l}\text { 环境变化和人类活动形成了新的生态位, 这些新生 } \\
\text { 态位更适合外来种生存。Anthropogenic changes } \\
\text { create novel niches, and these niches are better } \\
\text { exploited by exotic species. }\end{array}$ & $\begin{array}{l}\text { 生态位差异 } \\
\text { 和适合度差 } \\
\text { 异 Niche } \\
\text { and fitness } \\
\text { difference }\end{array}$ & $\begin{array}{l}\text { (1)由于外来种和土著种的生态位差异, 新 } \\
\text { 生态位只能被外来种占据。The novel } \\
\text { niches could only be occupied by exotics due } \\
\text { to the large exotic-native niche differences. } \\
\text { (2)外来种比土著种在新生态位中具有更高 } \\
\text { 的适合度。Exotics exhibit higher fitness than } \\
\text { natives in exploiting the new niches. }\end{array}$ & $\begin{array}{l}\text { Shea \& } \\
\text { Chesson, } \\
2002\end{array}$ \\
\hline
\end{tabular}

hypothesis)等强调外来种通过表型可塑性和适应性 进化等方式，使得自身在入侵地比原产地具有更高 的适合度，进而在同土著种的竞争中占据更大优 势。同时, 外部条件的变化也可以改变外来种的适 合度。例如, 在模拟氮沉降的情形下, 外来种适合 度提高的程度是要高于土著种的, 这可能是导致成 功入侵的关键因素(Vallano et al, 2012)。由于这类假 说聚焦适合度差异, 可以很好地解释为何竞争能力 强的外来种可以入侵成功并造成危害, 但在解释为 何竞争能力弱和危害程度小的外来种也可以大量定 居和归化上，则相对困难。

\section{3 综合考虑生态位和适合度差异的相关假说}

尽管很少有入侵假说同时考虑外来种和土著 种的生态位和适合度差异, 但是一些经典假说依然 可以在这个框架下进行解读。例如, 新生态位假说 (novel niche hypothesis)认为外来种可以更好地占据 由于环境变化和人类活动所形成的新生态位, 进而
实现入侵(Shea \& Chesson, 2002)。一方面, 我们可 以认为外来种和土著种存在较大生态位差异, 因此 新生态位只适合外来种生存。另一方面, 我们也可 以将该假说解读为: 在新生态位中外来种比土著种 具有更高的适合度，因此在竞争中占据优势。同样 地, 我们可以将天敌逃逸假说 (enemy release hypothesis)解读为: 因为天敌的丧失而导致外来种 在入侵地比原产地具有更高的繁殖成功率, 因此具 备更高的适合度。因此, 天敌逃逸假说本身强调了 适合度差异在入侵中的重要作用。同时, 天敌逃逸 假说也强调了外来种和土著种的天敌具有专一性, 专一性的天敌导致种内竞争大于种间竞争，通过负 密度制约和稀有种优势来维持外来种和土著种的 共存(Janzen-Connell假说; Petermann et al, 2008)。这 一稳定化机制对应的是外来种和土著种的生态位 差异。因此, 我们可以从现代物种共存理论出发考 虑天敌的影响, 无论是天敌逃逸导致的外来种适合 
度的提高, 还是外来种-土著种天敌的专一性导致 的生态位分化, 均能促进外来种的成功入侵。

通过同时考虑生态位差异和适合度差异, 我们 可以对经典入侵假说进行适当拓展和延伸, 发展和 丰富这些假说的内涵。例如新武器假说强调外来种 通过释放化感物质等方式来抑制土著种，进而提高 外来种相对于土著种的适合度优势实现入侵。如果 从生态位差异的角度出发, 外来物种能否通过“新 武器” 扩大自身和土著种的生态位差异, 进而实现 成功入侵呢? 对于干扰假说和波动资源假说而言, 干扰和资源的波动究竟是通过扩大外来种-土著种 的生态位差异还是提高外来种相对于土著种的适 合度优势来促进入侵的? 或者二者兼而有之? 生物 多样性对生物入侵的抵抗作用, 究竟是土著种的生 态位互补导致空余生态位的减少(互补效应, complementarity effect) 导致的, 还是多样性高的群 落有更大概率包含适合度高的土著种(取样效应, sampling effect)造成的(Fargione \& Tilman, 2005)? 全球变化因子和人类活动对生物入侵的促进作用, 究竟是通过为外来种创造合适的生态位, 还是提高 其适合度来实现的? 这些都是非常值得探讨的理 论问题。同样地, 这一理论框架也强调了当我们验 证竞争能力增强进化假说时, 不仅要比较外来种在 原产地和入侵地的竞争能力, 也应该比较入侵地外 来种和土著种的竞争能力(适合度), 只有外来种竞 争能力的提高程度可以实现外来种和土著种的共 存(图1中C区域至B区域的转变), 或者战胜土著种 时(图1中C区域至A区域的转变), 这一假说才能有 效地解释外来种成功入侵的机制。因此, 通过综合
考虑环境变化、人类活动和天敌等因素对外来种土著种生态位差异和适合度差异的影响, 现代物种 共存理论为整合经典入侵假说和发展新的入侵假 说提供了一个可操作的概念框架。

\section{3 外来种-土著种不同维度生态学差异的整会}

自Mark Davis和Daniel Simberloff的论战以来, 外来种和土著种是否存在生态学差异这一问题得 到了广泛关注。由于生态学差异较为抽象且难以量 化, 生态学家通常从外来种和土著种的亲缘关系和 性状差异的角度研究这一问题, 强调外来种在进化 历史和功能性状上的独特性, 以及外来种-土著种 的谱系距离和功能性状差异对入侵成功和危害的 影响(Leffler et al, 2014; Gallien \& Carboni, 2017)。这 些研究在深化我们对生物入侵机制理解的同时，却 也常常得出不一致的结论(Thompson \& Davis, 2011; Hulme \& Bernard-Verdier, 2018)。现代物种共存理论 可以通过综合考虑外来种-土著种的谱系距离、功能 性状差异、生态位差异和适合度差异这四者的相互 关系, 为解释目前不一致的研究结果提供一种新的 思路(图2)。

\section{1 外来种-土著种的谱系距离}

关于进化历史和谱系距离如何影响外来种的 归化和入侵, 达尔文在On the Origin of Species中提 出了截然相反的两个假说(Darwin, 1859)。一方面, 达尔文认为近缘物种之间存在着强烈的种间竞争， 土著种会抑制其近缘外来种的归化和入侵，因此和 土著种谱系距离远的外来种更容易入侵成功，该假 说被称为“达尔文归化假说” (Darwin’s naturalization

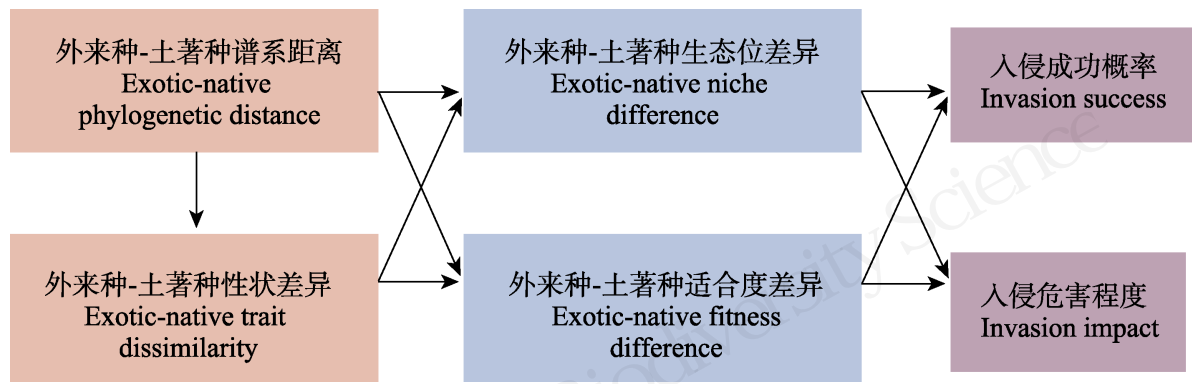

图2 外来种和土著种不同维度的生态学差异对生物入侵的影响。外来种和土著种的谱系距离会影响性状差异, 二者共同影 响外来种和土著种的生态位差异和适合度差异, 进而决定生物入侵的成功和危害。

Fig. 2 The effects of the ecological differences between exotics and natives on invasion outcome. Exotic-native phylogenetic distances influence their trait differences, which translate into exotic-native niche and/or fitness differences that combine to determine invasion success and impact. 
hypothesis; Daehler, 2001)。另一方面，达尔文认为 近缘物种偏好相似的生境, 因此土著种占据的生境 更适合其近缘外来种生存, 导致它们更容易成功定 居，该假说被称为 “预适应假说” (preadaptation hypothesis; Ricciardi \& Mottiar, 2006)。本质上, 这两 个对立的假说可以描述为“外来种-土著种的谱系距 离会抑制还是促进生物入侵”这一科学问题, 现在 综合在一起被称为“达尔文归化谜团” (Darwin’s naturalization conundrum, Diez et al, 2008)。近20年 来, 生态学家针对这一问题开展了大量的研究工作, 但是不同的研究往往得出相互矛盾的结论: 部分研 究支持预适应假说的预测, 部分研究支持达尔文归 化假说的预测, 也有部分研究没有得出明确的结论 (Ma et al, 2016; Cadotte et al, 2018)。这也是这一科 学问题被称为“谜团”的主要原因。尽管生态学家尝 试从空间尺度、入侵阶段和土著种灭绝等角度来解 释不同研究之间的分歧, 但该谜团的和解依旧存在 很大困难(Li et al, 2015a, b; Park et al, 2020)。

现代物种共存理论为该谜团的和解提供了一 个思路, 即达尔文的预测是否成立取决于外来种土著种谱系距离如何影响二者的生态位差异和适 合度差异(图2)。当谱系距离主要表征生态位差异时, 即近缘物种占据相似的生态位时, 与土著种亲缘关 系远的外来种更容易入侵, 符合达尔文归化假说的 预测。当谱系距离主要表征适合度差异时, 入侵的 结局取决于外来种和土著种适合度的相对大小。一 方面, 当外来种的适合度低于土著种时, 与土著种 谱系距离近的外来种和土著种适合度差异小, 更容 易入侵成功，符合预适应假说的预测；另一方面， 当外来种的适合度高于土著种时, 与土著种谱系距 离远的外来种适合度优势更大, 更容易入侵成功, 符合达尔文归化假说的预测。由于多个物种的谱系 距离与生态位和适合度差异可能存在复杂的关系, 谱系距离对生物入侵的影响可以是多变的。例如 $\mathrm{Li}$ 等(2019)利用细菌微宇宙实验发现, 相比谱系距离, 外来种-土著种的生态位差异和适合度差异可以更 好地预测外来种的入侵和危害程度。只有当谱系距 离可以很好地表征生态位差异时, 达尔文归化假说 的预测方能成立。尽管该研究结论的普遍性有待更 多的实验验证, 但这一研究清晰地表明了综合考虑 谱系距离、生态位差异和适合度差异的影响是破解
达尔文谜团的重要途径。

\section{2 外来种-土著种功能性状的差异}

在过去20多年里, 外来种和土著种功能性状的 比较一直是入侵生态学研究的热点话题 (van Kleunen et al, 2010b; Leffler et al, 2014)。生态学家也 一直在尝试从功能性状的角度预测潜在的入侵种 及其危害程度。该领域的研究通常遵循两种范式: 一是直接比较外来种及其亚类(如归化种、入侵种、 非入侵种、不同危害程度的外来种等)之间及其与土 著种在功能性状上的差别(Ordonez et al, 2010; van Kleunen et al, 2010a); 二是采用和达尔文归化谜团 类似的研究思路, 探讨外来种-土著种功能性状的 差异对生物入侵成功和危害的影响 (Gallien \& Carboni, 2017; Divíšek et al, 2018)。除了聚焦物种的 形态、生理、繁殖、遗传和行为等常规性状之外，物 种的资源利用效率、表型可塑性、遗传变异能力、 基因组大小和竞争能力等抽象性状也常被纳入比 较的范畴(Davidson et al, 2011; Suda et al, 2015; Zhang \& van Kleunen, 2019)。部分研究结果表明, 外来种尤其是入侵性强的外来种, 相比土著种通常 具有更快的生长速率、更高的叶片养分含量、更短 的世代周期、更小的基因组、将养分更多地分配给 生长生殖而非防御等性状特征(Ordonez et al, 2010; van Kleunen et al, 2010a; Suda et al, 2015)。但是也有 研究表明, 外来种和土著种在碳获取策略、生长速 率、竞争能力和繁殖力等性状上并没有显著不同 (Daehler, 2003; Leishman et al, 2010; Lemoine et al, 2016)。同样地, 关于功能性状差异如何影响生物入 侵的研究也呈现多元化的结果: 部分研究表明和土 著种功能性状相似的外来种更容易入侵, 部分研究 得出相反的结论, 也有研究发现外来种-土著种功 能性状的差异对生物入侵的影响在不同入侵阶段 是不同的(Escoriza \& Ruhí, 2016; Divíšek et al, 2018; El-Barougy et al, 2020)。总体上，外来种和土著种的 功能性状差异如何影响生物入侵, 依旧是一个充满 争议和不确定性的问题。

现代物种共存理论为外来种-土著种性状的比 较研究提供了一个新的思路, 即综合考虑性状差异 对生态位和适合度差异的影响。当物种的功能性状 主要表征生态位差异时，与土著种功能性状差异大 的外来种和土著种生态位重叠小, 因此更容易入 侵。当物种的功能性状主要表征适合度时, 则需要 
区分外来种和土著种的相对适合度大小。一方面, 当外来种的适合度低于土著种时, 具备和土著种相 似性状的外来种更容易入侵成功, 因为它们和土著 种的适合度差异更小。另一方面, 当外来种的适合 度高于土著种时，与土著种性状差异大的外来种更 容易入侵和造成危害, 因为它们具有更高的适合度 优势。因此, 笼统地讨论成功入侵的外来种是否具 有和土著种相似的性状, 以及和土著种具有相似性 状的外来种是否更难入侵等问题, 可能无法得出有 效的结论。在实际的群落中, 由于功能性状与生态 位和适合度差异存在复杂的相关关系, 需要针对不 同性状具体分析(Kraft et al, 2015)。例如, Godoy和 Levine (2014)以植物生长季高峰这一物候性状作为 研究对象, 发现外来种-土著种物候的差异同时影 响着生态位差异和适合度差异。一方面, 物候期差 异大的外来种-土著种组合具有更大的生态位差异, 促进了外来种在土著群落的定居; 另一方面, 物候 期晚的物种具有明显的适合度优势，在入侵过程中， 物候期晚的外来种可以战胜物候期早的土著种, 物 候期晚的土著种同样可以战胜物候期早的外来种。 总体上, 外来种和土著种物候期的时间差以及物候 期晚的竞争优势共同决定了入侵的成败。因此在开 展基于功能性状比较的生物入侵研究时, 必须全面 考虑生态位差异和适合度差异的影响。

\section{4 概念框架的局限性和研究展望}

现代物种共存理论在整合错综复杂的入侵假 说和诠释生态学差异如何影响生物入侵上展现了 强大的能力。但是和其他入侵生态学概念框架一样, 这一框架本身也存在一些缺陷和不足。如何弥补这 些不足, 并将这一框架更好地运用到不同生物入侵 的研究之中, 将是今后研究的重要方向。

\section{1 生态位差异和适合度差异的抽象性}

生态位差异和适合度差异是非常抽象的概念, 均不涉及具体的入侵机制, 而是对外来种和土著种 生态学差异的高度抽象和概括(储诚进等, 2017)。因 此在揭示某个具体的生物入侵事件时, 生态位差异 和适合度差异的比较能够提供的有效信息非常之 少。在群落生态学的概念框架中, Vellend (2016)将 选择、漂变、成种和扩散这四个抽象过程视为高层 次框架, 而将具体的群落构建假说(如中度干扰假 说、竞争排斥法则、种库假说、岛屿生物地理学理
论等)视为低层次过程(朱璧如和张大勇，2011)。类 似地, 我们可以将外来种-土著种的生态位差异和 适合度差异视为入侵生态学的高层次框架，而将具 体的入侵假说(如天敌逃逸假说、空余生态位假说和 竞争能力增强进化假说等)视为低层次机制。高层次 框架的目标在于简化和整合，以确保研究不同假说 的人员能够相互理解, 并尝试发展入侵生态学的普 适性理论。而低层次机制可以让我们深入理解特定 外来种在特定入侵地的明确入侵机制。从这个角度 而言, 基于现代物种共存理论的入侵生态学框架的 主要贡献在于为各种入侵假说的整合提供了一个 思路，使得假说之间的关系更加清晰，并有助于完 善其内在逻辑。但是当我们想揭示特定生物入侵事 件的运作方式和内在机制时，关注某个具体的假说 是合适且必要的。在实证研究中, 如何将现代物种 共存理论与具体的入侵假说有机地结合在一起，例 如探讨不同情形下外来种-土著种生态位和适合度 差异是由哪些机制决定的, 将是发展这一研究领域 的重要途径。

由于生态位差异和适合度差异的抽象性，如何 对其进行测度和量化一直是该领域的研究难点。 Godwin等(2020)综述了6种测度生态位差异和适合 度差异的方法, 但是这些方法需要拟合竞争系数、 内禀增长率和入侵增长率等复杂的种群动态参数, 在自然群落中难以展开。同时，由于生态位差异和 适合度差异并不独立, 物种间生态位差异的变化会 引起适合度差异的改变, 反之亦然(Song et al, 2019)。因此，比较二者在生物入侵中的相对贡献十 分困难。目前，基于现代物种共存理论的入侵生态 学研究十分匮乏, 仅有的实验研究也是在细菌微宇 宙和控制性草地等简化的模式系统中展开的(如 Godoy \& Levine, 2014; Li et al, 2019)。发展更加简 洁有效的生态位和适合度差异测度的替代方法, 并 将其运用到自然群落生物入侵的研究中, 将为该领 域的发展提供重要帮助。其中一个有益的尝试是用 外来种和土著种的性状距离的绝对值 (trait difference)来代表生态位差异，用外来种和土著种 性状的等级距离(trait hierarchy)来代表适合度差异 (如Carboni et al, 2016; Lai et al, 2015; Sheppard, 2019)。这些研究表明外来种可以通过扩大和土著种 的性状距离的绝对值(拥有和土著种差别大的性状) 和提高自身的性状等级(拥有比土著种优势的性状) 
两种策略实现成功入侵, 进而证实了现代物种共存 理论的主要预测。值得注意的是, 由于不同性状对 生态位和适合度差异的贡献是复杂多变的(Kraft et al, 2015), 这种方法只是对生态位差异和适合度差 异的近似度量, 发展更简单合理的度量方法依旧是 该领域的迫切需求。

\section{2 生态位差异和适合度差异的可变性}

物种的生态位和适合度不仅是物种本身的属 性, 也同时受到外部环境和其他物种的影响, 因此 外来种-土著种的生态位差异和适合度差异是高度 可变的。即使是相同的外来种-土著种组合, 在不同 的空间和时间中生态位差异和适合度差异也是不 同的。例如在空间异质性高的环境中, 外来种和土 著种可以占据不同的生境, 实现生态位分化, 具有 更高的生态位差异。同样地, 当环境具有高的时间 异质性时, 外来种和土著种可以通过储藏效应 (storage effect)提高彼此的生态位差异, 进而实现共 存。因此, 外部环境的变化会对外来种-土著种的生 态位差异和适合度差异产生重要影响。类似地, 外 来种自身的表型可塑性和适应性进化会导致其在 原产地和入侵地之间以及不同入侵地之间具有不 同的生态位和适合度。实际上, 增强竞争能力的进 化假说、氮分配的进化假说和新武器假说等假说本 身均强调了外来种在原产地和入侵地适合度的差 别。因此, 我们无法通过一个外来种在原产地的生 态位和适合度来预测其在入侵地的表现。这种变异 性给复杂的自然系统中外来种-土著种生态位和适 合度差异的度量带来了困难, 但也提供了很好的研 究机遇。其中一个值得探讨的科学问题是, 全球变 化和人类活动因子如何影响外来种-土著种的生态 位差异和适合度差异, 进而影响入侵的结局。例如 在温度升高、氮添加、生境片断化和人类干扰之后， 外来种和土著种究竟会提高生态位差异降低适合 度差异促进共存, 还是会降低生态位差异提高适合 度差异加剧竞争排除? 对这些问题开展控制性实 验验证将极大地丰富我们对全球变化和人类活动 背景下生物入侵机制的理解。

同样地, 外来种-土著种谱系距离和功能性状 对生态位差异和适合度差异的影响也并非一成不 变的(Hulme \& Bernard-Verdier, 2018)。例如, 即使谱 系距离可以很好地预测原产地的外来种和入侵地 的土著种的生态位差异和适合度差异, 外来种在入
侵地的适应性进化可以轻易地打破这种关系。功能 性状对物种生态位和适合度差异的影响在不同条 件下也会有显著的不同。例如, 在光资源充足的情 况下，树高这一性状可能更多地表征了植物间的生 态位差异，植物间树高的差异可以促进共存; 而在 光资源匮乏的情况下，树高可能主要表征植物间的 适合度差异，高的植物在竞争中占据优势，树高的 差异会导致竞争排除。因此，环境变化和人类活动 不仅会改变物种的性状、生态位和适合度本身，也 会改变这三者之间的相关关系。探讨在何种情形下 外来种-土著种谱系距离和功能性状可以很好地表 征其生态位差异和适合度差异, 以及哪些性状或性 状组合可以分别表征生态位差异和适合度差异, 将 是该领域的一个重要发展方向(Kraft et al, 2015; Li et al, 2019)。

\section{3 现代物种共存理论自身的发展}

自Chesson (2000)系统地提出现代物种共存理 论以来, 该理论自身也经历了一个不断发展和完善 的过程。早期的物种共存研究主要聚焦于成对物种 对资源的竞争，较少考虑多个物种之间复杂的相互 作用(如种间正相互作用和跨营养级的相互作用 等)。但是在生物入侵过程中, 种间正相互作用和跨 营养级的相互作用是非常普遍的。比如在一个外来 种成功入侵之后, 可能会促进其他外来种后续的入 侵，进而放大入侵的危害(入侵崩溃假说，invasional meltdown hypothesis; Simberloff \& Von Holle, 1999)。同时，一个外来种成功入侵之后，也可能通 过优先级效应(priority effects; Fukami, 2015)抑制其 他外来种的入侵。跨营养级的相互作用, 如食物网 (王少鹏, 2020)和传粉网络(Valdovinos et al, 2018)的 结构和功能, 也会对入侵产生复杂的影响。因此, 在自然群落中, 多个外来种和多个土著种可以产生 复杂的相互作用。尽管我们可以通过度量外来种和 土著种的平均生态位差异和平均适合度来开展研 究(例如将入侵地的土著种群落当成一个物种来对 待), 但是这种简化处理的方式无法应对复杂的种 间相互关系。例如三个物种的竞争可能存在石头一 剪刀-布的关系(rock-paper-scissors competitive loop), 亦或第三个物种可以通过影响两个物种的相互作 用进而影响共存 (高阶相互作用, higher-order interaction) (Levine et al, 2017; 李远智等, 2020)。可 喜的是, 现代物种共存理论在近些年取得了突飞猛 
进的发展, 不同生态学家通过不同的方法陆续把种 间正相互作用、天敌逃逸、优先级效应以及多个物 种多个营养级的相互作用纳入到现代物种共存理 论的概念范畴之中(Chesson, 2018; Ellner et al, 2019; Grainger et al, 2019a; Ke \& Wan, 2020)。例如, Godoy (2019)建议以现代物种共存理论为基础, 通过考虑 多物种和多营养级网络的结构稳定性(宋础良，2020) 的方式, 发展生物入侵的研究框架。尽管目前这些 整合还存在着广泛的争论, 远没有达到统一和完善 的地步, 但这些新思想和新方法的建立无疑将深化 我们对物种共存的理解(Barabás et al, 2018), 其在 入侵生态学中的推广和应用也将进一步发展生物 入侵机制的相关理论。

同时, 有关生物入侵的研究一直在现代物种共 存理论的发展中占据重要地位。一方面, 相互入侵 准则(mutual invasion criterion)一直是判断物种能否 稳定共存的重要标准(Grainger et al, 2019b)。该准则 认为, 当物种以低密度入侵由其竞争者构成的稳定 群落时, 如果每个物种均能实现正的种群增长率, 则物种间可以稳定共存。依据这一准则, 我们可以 通过物种间的相互入侵实验，量化物种间的生态位 差异和适合度差异, 进而比较二者在物种共存中的 相对贡献(Carroll et al, 2011; Godoy \& Levine, 2014)。由于相互入侵准则不局限于成对物种, 这一 实验思路可以综合考虑优先级效应、种间正相互作 用、高阶相互作用和跨营养级的作用, 促进了现代 物种共存理论在性状替换(character displacement)、 物种分布区、气候变化等多个领域的应用和发展 (Grainger et al, 2019b)。另一方面, 在全球生物入侵 日益严峻的新形势下, 具有不同进化历史和长期地 理隔离的外来种和土著种发生了新的种间相互作 用, 组成了新型生态系统(novel ecosystems), 为我 们研究物种共存理论提供了天然的实验室。利用自 然生物入侵过程作为模式系统来研究现代物种共 存理论, 很可能将是发展和完善该理论的重要途径。

\section{5 结语}

外来种和土著种是否具有生态学差异, 以及这 种差异如何影响外来种入侵, 一直是入侵生态学研 究和争论的核心问题。现代物种共存理论从生态位 和适合度差异的角度出发, 有望将那些看似数不清 且难以理解的入侵假说联系起来, 使其变得易于理
解且逻辑连贯。这一理论可以将外来种-土著种的谱 系距离、功能性状、生态位差异和适合度差异综合 考虑，进而建立一个统一的外来种-土著种比较框 架。该概念框架与基于群落构建理论的框架 (Kiinunen et al, 2016; Pearson et al, 2018)、基于入侵 阶段划分的框架(Blackburn et al, 2011)、基于进化和 生态综合的框架(Facon et al, 2006)等一道，在体现 入侵生态学的复杂性、多元性和整体性的同时，共 同为发展和完善入侵生态学的学科体系指明了方 向。最近 10 年, 现代物种共存理论处于快速发展的 时期，新理论和新方法不断涌现 (Barabás et al, 2018)。但是这一理论在入侵生态学中的运用和实证 尚处于起步阶段，研究思路和方法均未成熟。这对 于我们而言，既是挑战也是机遇。希望通过本文的 粗浅介绍, 抛砖引玉，吸引我国的生态学家更多地 关注这一理论问题, 将现代物种共存理论更多地运 用到生物入侵的研究之中。

致谢: 感谢张大勇老师和王少鹏老师的约稿和审稿 人提供的宝贵修改意见。

\section{参考文献}

Adler PB, HilleRisLambers J, Levine JM (2007) A niche for neutrality. Ecology Letters, 10, 95-104.

Baker HG (1974) The evolution of weeds. Annual Review of Ecology and Systematics, 5, 1-24.

Barabás G, D’Andrea R, Stump SM (2018) Chesson's coexistence theory. Ecological Monographs, 88, 277-303.

Blackburn TM, Pyšek P, Bacher S, Carlton JT, Duncan RP, Jarošík V, Wilson JRU, Richardson DM (2011) A proposed unified framework for biological invasions. Trends in Ecology and Evolution, 26, 333-339.

Blossey B, Notzold R (1995) Evolution of increased competitive ability in invasive nonindigenous plants: A hypothesis. Journal of Ecology, 83, 887-889.

Cadotte MW, Campbell SE, Li SP, Sodhi DS, Mandrak NE (2018) Preadaptation and naturalization of nonnative species: Darwin's two fundamental insights into species invasion. Annual Review of Plant Biology, 69, 661-684.

Callaway RM, Aschehoug ET (2000) Invasive plants versus their new and old neighbors: A mechanism for exotic invasion. Science, 290, 521-523.

Carboni M, Münkemüller T, Lavergne S, Choler P, Borgy B, Violle C, Essl F, Roquet C, Munoz F, Consortium D, Thuiller W (2016) What it takes to invade grassland ecosystems: Traits, introduction history and filtering processes. Ecology Letters, 19, 219-229.

Carroll IT, Cardinale BJ, Nisbet RM (2011) Niche and fitness 
differences relate the maintenance of diversity to ecosystem function. Ecology, 92, 1157-1165.

Catford JA, Jansson R, Nilsson C (2009) Reducing redundancy in invasion ecology by integrating hypotheses into a single theoretical framework. Diversity and Distributions, 15, 22-40.

Chesson P (2000) Mechanisms of maintenance of species diversity. Annual Review of Ecology and Systematics, 31, 343-366.

Chesson P (2018) Updates on mechanisms of maintenance of species diversity. Journal of Ecology, 106, 1773-1794.

Chu CJ, Adler PB (2015) Large niche differences emerge at the recruitment stage to stabilize grassland coexistence. Ecological Monographs, 85, 373-392.

Chu CJ, Wang YS, Liu Y, Jiang L, He FL (2017) Advances in species coexistence theory. Biodiversity Science, 25, 345-354. (in Chinese with English abstract) [储诚进, 王西 石, 刘宇, 蒋林, 何芳良 (2017) 物种共存理论研究进展. 生物多样性, 25, 345-354.]

Daehler CC (2001) Darwin's naturalization hypothesis revisited. The American Naturalist, 158, 324-330.

Daehler CC (2003) Performance comparisons of co-occurring native and alien invasive plants: Implications for conservation and restoration. Annual Review of Ecology, Evolution, and Systematics, 34, 183-211.

Darwin C (1859) On the Origin of Species. John Murray, London.

Davidson AM, Jennions M, Nicotra AB (2011) Do invasive species show higher phenotypic plasticity than native species and, if so, is it adaptive? A meta-analysis. Ecology Letters, 14, 419-431.

Davis MA, Grime JP, Thompson K (2000) Fluctuating resources in plant communities: A general theory of invasibility. Journal of Ecology, 88, 528-534.

Davis MA (2009) Invasion Biology. Oxford University Press, Oxford.

Davis MA, Chew MK, Hobbs RJ, Lugo AE, Ewel JJ, Vermeij GJ, Brown JH, Rosenzweig ML, Gardener MR, Carroll SP, Thompson K, Pickett STA, Stromberg JC, Del Tredici P, Suding KN, Ehrenfeld JG, Grime JP, Mascaro J, Briggs JC (2011) Don't judge species on their origins. Nature, 474, 153-154.

Diez JM, Sullivan JJ, Hulme PE, Edwards G, Duncan RP (2008) Darwin's naturalization conundrum: Dissecting taxonomic patterns of species invasions. Ecology Letters, 11, 674-681.

Divíšek J, Chytrý M, Beckage B, Gotelli NJ, Lososová Z, Pyšek P, Richardson DM, Molofsky J (2018) Similarity of introduced plant species to native ones facilitates naturalization, but differences enhance invasion success. Nature Communications, 9, 4631.

Drake JA, Mooney HA, di Castri F, Groves RH, Kruger FJ, Rejmánek M, Williamson M (1989) Biological Invasions: A
Global Perspective. Wiley, Chichester.

El-Barougy RF, Elgamal I, Rohr RP, Probert AF, Abdel-hamid AK, Bacher S (2020) Functional similarity and dissimilarity facilitate alien plant invasiveness along biotic and abiotic gradients in an arid protected area. Biological Invasions, 22, 1997-2016.

Ellner SP, Snyder RE, Adler PB, Hooker G (2019) An expanded modern coexistence theory for empirical applications. Ecology Letters, 22, 3-18.

Elton CS (1958) The Ecology of Invasions by Animals and Plants. Chapman and Hall, London.

Escoriza D, Ruhí A (2016) Functional distance to recipient communities may favour invasiveness: Insights from two invasive frogs. Diversity and Distributions, 22, 519-533.

Facon B, Genton BJ, Shykoff J, Jarne P, Estoup A, David P (2006) A general eco-evolutionary framework for understanding bioinvasions. Trends in Ecology and Evolution, 21, 130-135.

Fargione JE, Tilman D (2005) Diversity decreases invasion via both sampling and complementarity effects. Ecology Letters, 8, 604-611.

Feng YL, Lei YB, Wang RF, Callaway RM, Valiente-Banuet A, Inderjit, Li YP, Zheng YL (2009) Evolutionary tradeoffs for nitrogen allocation to photosynthesis versus cell walls in an invasive plant. Proceedings of the National Academy of Sciences, USA, 106, 1853-1856.

Fukami T (2015) Historical contingency in community assembly: Integrating niches, species pools, and priority effects. Annual Review of Ecology, Evolution, and Systematics, 46, 1-23.

Gallien L, Carboni M (2017) The community ecology of invasive species: Where are we and what's next? Ecography, 40, 335-352.

Godoy O, Levine JM (2014) Phenology effects on invasion success: Insights from coupling field experiments to coexistence theory. Ecology, 95, 726-736.

Godoy O (2019) Coexistence theory as a tool to understand biological invasions in species interaction networks: Implications for the study of novel ecosystems. Functional Ecology, 33, 1190-1201.

Godwin CM, Chang FH, Cardinale BJ (2020) An empiricist's guide to modern coexistence theory for competitive communities. Oikos, 129, 1109-1127.

Grainger TN, Letten AD, Gilbert B, Fukami T (2019a) Applying modern coexistence theory to priority effects. Proceedings of the National Academy of Sciences, USA, 116, 6205-6210.

Grainger TN, Levine JM, Gilbert B (2019b) The invasion criterion: A common currency for ecological research. Trends in Ecology and Evolution, 34, 925-935.

Hulme PE, Bernard-Verdier M (2018) Comparing traits of native and alien plants: Can we do better? Functional Ecology, 32, 117-125. 
Ju RT, Li H, Shih CJ, Li B (2012) Progress of biological invasions research in China over the last decade. Biodiversity Science, 20, 581-611. (in Chinese with English abstract) [鞠瑞亭, 李慧, 石正人, 李博 (2012) 近十年中 国生物入侵研究进展. 生物多样性, 20, 581-611.]

Ke PJ, Wan J (2020) Effects of soil microbes on plant competition: A perspective from modern coexistence theory. Ecological Monographs, 90, e01391.

Keane RM, Crawley MJ (2002) Exotic plant invasions and the enemy release hypothesis. Trends in Ecology and Evolution, 17, 164-170.

Kinnunen M, Dechesne A, Proctor C, Hammes F, Johnson D, Quintela-Baluja M, Graham D, Daffonchio D, Fodelianakis S, Hahn N, Boon N, Smets BF (2016) A conceptual framework for invasion in microbial communities. The ISME Journal, 10, 2773-2779.

Kraft NJB, Godoy O, Levine JM (2015) Plant functional traits and the multidimensional nature of species coexistence. Proceedings of the National Academy of Sciences, USA, 112, 797-802.

Lai HR, Mayfield MM, Gay-des-combes JM, Spiegelberger T, Dwyer JM (2015) Distinct invasion strategies operating within a natural annual plant system. Ecology Letters, 18, 336-346.

Lawton JH (1999) Are there general laws in ecology? Oikos, 84, 177-192.

Leffler AJ, James JJ, Monaco TA, Sheley RL (2014) A new perspective on trait differences between native and invasive exotic plants. Ecology, 95, 298-305.

Leishman MR, Thomson VP, Cooke J (2010) Native and exotic invasive plants have fundamentally similar carbon capture strategies. Journal of Ecology, 98, 28-42.

Lemoine NP, Burkepile DE, Parker JD (2016) Quantifying differences between native and introduced species. Trends in Ecology and Evolution, 31, 372-381.

Levine JM, D’Antonio CM (1999) Elton revisited: A review of evidence linking diversity and invasibility. Oikos, 87, 15-26.

Levine JM, Bascompte J, Adler PB, Allesina S (2017) Beyond pairwise mechanisms of species coexistence in complex communities. Nature, 546, 56-64.

Li SP, Cadotte MW, Meiners SJ, Hua ZS, Shu HY, Li JT, Shu WS (2015a) The effects of phylogenetic relatedness on invasion success and impact: Deconstructing Darwin's naturalization conundrum. Ecology Letters, 18, 1285-1292.

Li SP, Guo T, Cadotte MW, Chen YJ, Kuang JL, Hua ZS, Zeng Y, Song Y, Liu Z, Shu WS, Li JT (2015b) Contrasting effects of phylogenetic relatedness on plant invader success in experimental grassland communities. Journal of Applied Ecology, 52, 89-99.

Li SP, Tan J, Yang X, Ma C, Jiang L (2019) Niche and fitness differences determine invasion success and impact in laboratory bacterial communities. The ISME Journal, 13,
402-412.

Li YZ, Xiao JL, Liu HL, Wang YS, Chu CJ (2020) Advances in higher-order interactions between organisms. Biodiversity Science, 28, 1333-1344. (in Chinese with English abstract) [李远智, 肖俊丽, 刘翰伦, 王西石, 储诚进 (2020) 生物 间高阶相互作用研究进展. 生物多样性, 28, 1333-1344.]

Lockwood JL, Cassey P, Blackburn T (2005) The role of propagule pressure in explaining species invasions. Trends in Ecology and Evolution, 20, 223-228.

Lockwood JL, Hoopes MF, Marchetti MP (2007) Invasion Ecology. Blackwell Publishing, Malden.

Lowry E, Rollinson EJ, Laybourn AJ, Scott TE, Aiello-Lammens ME, Gray SM, Mickley J, Gurevitch J (2013) Biological invasions: A field synopsis, systematic review, and database of the literature. Ecology and Evolution, 3, 182-196.

Lozon JD, MacIsaac HJ (1997) Biological invasions: Are they dependent on disturbance? Environmental Reviews, 5, 131-144.

Ma C, Li SP, Pu ZC, Tan JQ, Liu MQ, Zhou J, Li HX, Jiang L (2016) Different effects of invader-native phylogenetic relatedness on invasion success and impact: A meta-analysis of Darwin's naturalization hypothesis. Proceedings of the Royal Society B: Biological Sciences, 283, 20160663.

MacDougall AS, Gilbert B, Levine JM (2009) Plant invasions and the niche. Journal of Ecology, 97, 609-615.

Melbourne BA, Cornell HV, Davies KF, Dugaw CJ, Elmendorf S, Freestone AL, Hall RJ, Harrison S, Hastings A, Holland M, Holyland M, Holyoak M, Lambrinos J, Moore K, Yokomizo H (2007) Invasion in a heterogeneous world: Resistance, coexistence or hostile takeover? Ecology Letters, 10, 77-94.

Mooney HA, Drake JA (1986) Ecology of Biological Invasions of North America and Hawaii. Springer, New York.

Ordonez A, Wright IJ, Olff H (2010) Functional differences between native and alien species: A global-scale comparison. Functional Ecology, 24, 1353-1361.

Park DS, Feng X, Maitner BS, Ernst KC, Enquist BJ (2020) Darwin's naturalization conundrum can be explained by spatial scale. Proceedings of the National Academy of Sciences, USA, 117, 10904-10910.

Pearson DE, Ortega YK, Eren Ö, Hierro JL (2018) Community assembly theory as a framework for biological invasions. Trends in Ecology and Evolution, 33, 313-325.

Petermann JS, Fergus AJF, Turnbull LA, Schmid B (2008) Janzen-Connell effects are widespread and strong enough to maintain diversity in grasslands. Ecology, 89, 23992406.

Pettitt-Wade H, Wellband KW, Heath DD, Fisk AT (2015) Niche plasticity in invasive fishes in the Great Lakes. Biological Invasions, 17, 2565-2580.

Pyšek P, Hulme PE (2009) Invasion biology is a discipline that's too young to die. Nature, 460, 324-324. 
Ricciardi A, Mottiar M (2006) Does Darwin's naturalization hypothesis explain fish invasions? Biological Invasions, 8, 1403-1407.

Richardson DM, Pyšek P (2006) Plant invasions: Merging the concepts of species invasiveness and community invasibility. Progress in Physical Geography: Earth and Environment, 30, 409-431.

Richardson DM, Ricciardi A (2013) Misleading criticisms of invasion science: A field guide. Diversity and Distributions, 19, 1461-1467.

Shea K, Chesson P (2002) Community ecology theory as a framework for biological invasions. Trends in Ecology and Evolution, 17, 170-176.

Sheppard CS (2019) Relative performance of co-occurring alien plant invaders depends on traits related to competitive ability more than niche differences. Biological Invasions, 21, 1101-1114.

Shigesada N, Kawasaki K (1997) Biological Invasions: Theory and Practice. Oxford University Press, Oxford.

Simberloff D, Von Holle B (1999) Positive interactions of nonindigenous species: Invasional meltdown? Biological Invasions, 1, 21-32.

Simberloff D (2011) Non-natives: 141 scientists object. Nature, 475, 36.

Simberloff D, Vitule JRS (2014) A call for an end to calls for the end of invasion biology. Oikos, 123, 408-413.

Simberloff D (2015) Non-native invasive species and novel ecosystems. F1000prime Reports, 7, 47.

Song C, Barabás G, Saavedra S (2019) On the consequences of the interdependence of stabilizing and equalizing mechanisms. The American Naturalist, 194, 627-639.

Song CL (2020) Structural stability: Concepts, methods, and applications. Biodiversity Science, 28, 1345-1361. (in Chinese with English abstract) [宋础良 (2020) 结构稳定 性：概念、方法和应用. 生物多样性, 28, 1345-1361.]

Suda J, Meyerson LA, Leitch IJ, Pyšek P (2015) The hidden side of plant invasions: The role of genome size. New Phytologist, 205, 994-1007.

Thompson K, Davis MA (2011) Why research on traits of invasive plants tells us very little. Trends in Ecology and Evolution, 26, 155-156.

Valdovinos FS, Berlow EL, de Espanés PM, Ramos-Jiliberto R, Vázquez DP, Martinez ND (2018) Species traits and network structure predict the success and impacts of pollinator invasions. Nature Communications, 9, 2153.

Valéry L, Fritz H, Lefeuvre JC (2013) Another call for the end of invasion biology. Oikos, 122, 1143-1146.

Vallano DM, Selmants PC, Zavaleta ES (2012) Simulated nitrogen deposition enhances the performance of an exotic grass relative to native serpentine grassland competitors. Plant Ecology, 213, 1015-1026. van Kleunen M, Dawson W, Schlaepfer D, Jeschke JM, Fischer M (2010a) Are invaders different? A conceptual framework of comparative approaches for assessing determinants of invasiveness. Ecology Letters, 13, 947-958.

van Kleunen M, Weber E, Fischer M (2010b) A meta-analysis of trait differences between invasive and non-invasive plant species. Ecology Letters, 13, 235-245.

Vellend M (2016) The Theory of Ecological Communities. Princeton University Press, Princeton and Oxford.

Wan FH, Hou YM, Jiang MX (2015) Invasion Biology. Science Press, Beijing. (in Chinese) [万方浩, 侯有明, 蒋 明星 (2015) 入侵生物学. 科学出版社, 北京.]

Wang MN, Dai ZC, Qi SS, Wang XY, Du DL (2014) Main hypotheses and research progress of invasive mechanism of alien plants. Jiangsu Agricultural Sciences, 42(12), 378-382. (in Chinese) [王明娜, 戴志聪, 祁珊珊, 王晓莹, 杜道林 (2014) 外来植物入侵机制主要假说及其研究进展. 江苏 农业科学, 42(12), 378-382.]

Wang SP (2020) Food web structure and functioning: Theoretical advances and outlook. Biodiversity Science, 28, 1391-1404. (in Chinese with English abstract) [王少鹏 (2020) 食物网结构与功能: 理论进展与展望. 生物多样 性, 28, 1391-1404.]

Williamson M (1996) Biological Invasions. Springer, New York.

Wu H, Ding JQ (2014) Recent progress in invasion ecology. Chinese Science Bulletin, 59, 438-448. (in Chinese with English abstract) [吴昊, 丁建清 (2014) 入侵生态学最新 研究动态. 科学通报, 59, 438-448.]

Xu CY, Zhang WJ, Lu BR, Chen JK (2001) Progress in studies on mechanisms of biological invasion. Biodiversity Science, 9, 430-438. (in Chinese with English abstract) [徐承远, 张 文驹, 卢宝荣, 陈家宽 (2001) 生物入侵机制研究进展. 生物多样性, 9, 430-438.]

Xu RM, Ye WH (2003) Biological Invasions: Theory and Practice. Science Press, Beijing. (in Chinese) [徐汝梅, 叶万 辉 (2003) 生物入侵理论与实践. 科学出版社, 北京.]

Zhang ZJ, van Kleunen M (2019) Common alien plants are more competitive than rare natives but not than common natives. Ecology Letters, 22, 1378-1386.

Zheng JM, Ma KP (2010) Invasion Ecology. Higher Education Press, Beijing. (in Chinese) [郑景明, 马克平 (2010) 入侵 生态学. 高等教育出版社, 北京.]

Zhu BR, Zhang DY (2011) A process-based theoretical framework for community ecology. Biodiversity Science, 19, 389-399. (in Chinese with English abstract) [朱璧如, 张大勇 (2011) 基于过程的群落生态学理论框架. 生物 多样性, 19, 389-399.]

(责任编委: 张大勇 责任编辑: 问文杰) 